\title{
MEDICAL IMAGE COMPRESSION TECHNIQUE USING LISTLESS SET PARTITIONING IN HIERARCHICAL TREES AND CONTEXTUAL VECTOR QUANTIZATION FOR BRAIN IMAGES
}

\author{
${ }^{1}$ Sridevi, S. and ${ }^{2}$ V.R. Vijayakumar \\ ${ }^{1}$ Department of Computer Science and Engineering, Sethu Insitute of Technology, Kariapatti, Tamil Nadu, India \\ ${ }^{2}$ Department of Electronics and Communication Engineering, Anna University, Coimbatore, Tamil Nadu, India
}

Received 2013-07-06, Revised 2013-07-24; Accepted 2013-08-03

\begin{abstract}
A hybrid image compression techniques has been developed to compress medical images. Due to the extensive use of medical images like CT and MR scan, these medical imagery are stored for a longer period for the continuous monitoring of the patients and the amount of data associated with images is large and it occupies enormous storage capacity. So, the medical images need to be compressed to reduce the storage cost and for transmission without any loss. In this study, a hybrid method which combines the Listless Set Partitioning in Hierarchical Trees (LSPIHT) and the Contextual Vector Quantization (CVQ) method for the compression of brain images. Here, the region containing the most important information for diagnosis is called Region of Interest (ROI) and this is to be compressed with out any loss in the quality. In this method, the ROI is encoded separately using LSPIHT and the Back Ground region (BG) is encoded using CVQ. Finally, the two regions are merged together to get the reconstructed image. Our results show that the proposed method gives very good image quality for diagnosis without any degradable loss. The performance of the compression technique is evaluated using the parameters (CR, MSE, PSNR) and achieved better result compared to other existing methods. As a result, we strongly believe that using our method, we can overcome the limitations in storage and transmission of medical images that are produced day by day.
\end{abstract}

Keywords: Lspiht, CVQ, ROI, BG, MSE, CR, PSNR

\section{INTRODUCTION}

Image compression techniques play a major in all areas, especially in medical image domain. The medical images are stored for a longer time and it has to be transmitted to other location like from one hospital to another hospital for reference. To store and transmit these medical images, we need enormous amount of storage capacity and bandwidth. Due to the limitations of storage and transmission, the medical images are to be compressed. Normally, compression can be classified into two major categories. (1) Lossy compression and (2) Lossless compression. In lossy compression, there will be a small distortion in the image with higher compression rate. But in the case of lossless, the image is compressed without any loss in the quality.

Recent study shows that there has been great interest in lossy compression of medical images. Here the loss in data are very minimal and there wont be any degradable loss in the quality of image for diagnosis by the radiologists. Nowadays, lossy compression techniques are combined with scalar or vector quantization for efficient compression result.

Many compression algorithms (Gonzalez and Woods, 2009) produce high compression rates with affordable loss of quality but physician may not accept any loss in diagnostically important regions of images. The region which contains the more important information Corresponding Author: Sridevi, S., Department of Computer Science and Engineering, Sethu Insitute of Technology, Kariapatti, Tamil Nadu, India 
for diagnosis is referred to as region of interest (Selvi and Nadarajan, 2012). The main challenge in compressing medical images is to compress the ROI region without any loss of important information.

In our work, we propose new technique based on ROI and vector quantization ( $\mathrm{Lu}$ and Chang, 2010). To achieve better compression rate, we combine Listless SPIHT (Alam and Khan, 2012) with contextual vector quantization.

The ROI region and the background regions are encoded separately and the two encoded images are merged to get a compressed image.

The outline of this study is as follows; Section 2 describes the material and methods used and the Section 3 deals with the results and discussion. Conclusion and Acknowledgement, references are presented in Section 4,5 and 6 , respectively.

\subsection{Related Works}

Vector quantization for lossy image compression is a widely accepted method and it is applied to different image compression schemes. The main aim of this VQ is to generate code book which is used to represent the image data. In VQ, the image is divided into $\mathrm{n}$ dimension training vectors. Based on the clustering method, code book is generated and the index of the code vector with lowest distortion is obtained from the encoder during encoding process. Lowest distortion is calculated using the Euclidian distance. That is the lowest Euclidian distance between the input vector and each code vector in the code book. In the decoding process the index of the code vector is searched in the code book and the searched code vector is used for the reconstruction of the image.

For ROI coding, JPEG2K is the first method that uses a scaling method of coding. In this method, ROI shape is limited to circles and rectangles. The scaling methods lead to two major problems in encoding. First, it needs to encode the ROI shape and if the shape is arbitrary then coding will consume more no of bits which minimizes the efficiency of coding.

Another method for ROI is the Maxshift method (Sanchez et al., 2010), which uses the scaling factor 2 s, where $s$ is the non-all-zero bit plane. A binary shape mask with more coefficients than pixels in the image ROI is formed. After decoding, the transform ROI consists of coefficients with magnitude greater than $2 \mathrm{~s}$ and these are scaled down by $2 \mathrm{~s}$. This method lacks in flexibility in scaling value and overflow of bit stream.

Embedded Block coding is another ROI based method, (Grailu et al., 2013) the main advantage of these method is to get a target compression ratio and need not perform multiple compression. But this method suffers from very high computational complexity.

Recently, (Hosseini and Naghsh-Nilchi, 2012) proposed a technique based on Contextual vector quantization for CT image compression.

In our work, we propose a similar ROI based hybrid technique for the compression of Magnetic Resonance Images which uses Listless SPIHT and Contextual Vector Quantization.

\subsection{ROI Based Coding Techniques 1.2.1. JPEG2K (Scaling Method)}

In JPEG2K (Bartrina-Rapesta et al., 2011), ROI coding is the scaling method in which the wavelet coefficients belonging to the contextual regions are shifted upward. Moreover this method allows the use of arbitrary scaling value. But it has some drawbacks. First, the shape information of the ROI needs to be encoded. Second, if arbitrary ROI shapes (Seo et al., 2010) are selected then the coding will consume more number of bits which in turn decreases the overall efficiency of coding.

\subsection{EBCOT Coding}

In this method, the input image is segmented into two region called foreground and the background, respectively and shape adaptive Integer Wavelet Transform (IWT) (Thorat and Jadhav, 2010) for lossy to lossless is applied to both the regions separately. Finally, the two bit streams (foreground and the BG) are merged into a single bit stream. Here, ROI mask is generated and it is used to identify the ROI coefficients.

\subsection{Maxshift}

This method (defined in JPEG 2K-part 1) uses the arbitrary scaling value without the need for transmitting the shape information to the decoder. Here, the mapping is based on the wavelet filters which maps the ROI from the spatial domain to the wavelet domain. Wavelet coefficients that are not part of ROI are scaled down. The main advantage of this method is that encoding of arbitrarily shaped ROI is possible without the shape information.

\subsection{Implicit ROI Coding}

This method (Lim et al., 2010) is more advantageous than the Maxshift method. For encoding and decoding, no bit plane scaling is needed and it is very easy to implement because of its low complexity. Priority is assigned on the basis of block by block. 


\subsection{SPIHT and Contextual SPIHT}

Set Partitioning in Hierarchical Trees algorithm (SPIHT), (Singh and Singh, 2012) uses parent child relationship among the wavelet coefficients and a tree structure is formed using the coefficients those who are in same spatial area.

Contextual SPIHT was introduced by (Ansari and Anand, 2009), to compress ultra sound images. This method uses SPIHT algorithm, to separate the coefficients. Here an interactive segmentation approach is used to separate ROI (Noma et al., 2012) and not the $\mathrm{BG}$ of the image and the two regions are encoded separately using CSPIHT with high bitrate and low bitrate respectively. Finally the two regions are merged to reconstruct the image.

\subsection{Contextual Vector Quantization}

This method (Hosseini and Naghsh-Nilchi, 2012) is also based on Contextual SPIHT method. Here instead of SPIHT, Vector Quantization is used to encode the image parts. Figure 1 shows the block diagram of vector quantization. The main aim of this approach is to separate ROI and Background (BG) and then encode both regions using $\mathrm{CVG}$. Here the ROI is encoded with high bit rate and the $\mathrm{BG}$ is encoded with low bit rate. Finally, the two images are merged to get the compressed image.

\section{MATERIALS AND METHODS}

\subsection{Proposed Hybrid Listless SPIHT and CVQ}

A novel wavelet based compression method is proposed which uses a ROI technique to extract the important information and back ground image. In this, the diagnostically Important Region (ROI) and the BG are encoded separately using Listless SPIHT (LSPIHT) and Contextual Vector Quantization (CVQ). The ROI is extracted using connected component labeling method and encoded separately using LSPIHT with high bpp and the BG is encoded using CVQ with low bpp. Finally, the two encoded regions are merged together to get the compressed image. The flow diagram of the proposed method is shown in Fig. 2.

Listless Set Partitioning in Hierarchical Trees (LSPIHT) uses the set structure and partitioning rules. It uses a set partitioning rules of SPIHT without using any list. In the listless SPIHT, the wavelet coefficients are arranged in recursive $\mathrm{z}$ ordering with single linear index and the indexing is used to track the set partitioning. To store the significant and insignificant coefficients, state markers are used. Markers are used for each pixel to identify insignificant/significant and newly significant coefficients and there are five markers to mark the state of sets as well as to denote the spatial resolution level of these sets.

Contextual Vector Quantization (CVQ) is used to encode the background image with low bpp. Here the vector quantization principle is followed to generate code book or code words. LBG algorithm is based on VQ. The algorithm is: (1) Code book is generated using splitting method. (2) Initial code vector is created by computing the average of training vectors (3) code vector is splitted into two code vectors using a distortion threshold constant (4) Euclidean distance is used to cluster the training set vectors.

The Euclidean distance is defined Equation 1:

$d\left(x, y_{i}\right)=\sqrt{\sum_{j=1}^{k}\left(x_{j}-y_{i j}\right)^{2}}$

where, $x_{j}$ is the $j^{\text {th }}$ component of the training set vector the $\mathrm{j}^{\text {th }}$ component of the code-vector $\mathrm{y}_{\mathrm{i}}$.

Clusters average is calculated using this algorithm to form a vector which is replaced with the code vector. Cluster average is calculates as Equation 2:

$\mathrm{y}_{\mathrm{i}}=\frac{1}{\mathrm{~m}} \sum_{\mathrm{j}=1}^{\mathrm{m}} \mathrm{x}_{\mathrm{ij}}$

where, $\mathrm{i}$ is the component of each vector and $\mathrm{m}$ is the vectors in the cluster. Then the average distortions for the entire code book is calculated using the formula Equation 3:

$\mathrm{D}_{\mathrm{ave}}^{(\mathrm{i})}=\frac{1}{\mathrm{M} \cdot \mathrm{K}} \sum_{\mathrm{i}=1}^{\mathrm{M}}\left\|\mathrm{x}_{\mathrm{i}}-\mathrm{Q}\left(\mathrm{x}_{\mathrm{i}}\right)\right\|^{2}$

where, $\mathrm{M}$ is the size of training set, $\mathrm{k}$ is the size of each vector $\mathrm{Q}(\mathrm{x})$ is the nearest vector to vector $\mathrm{x}$ and \|\| is a symbol for Euclidean distance.

\subsection{Segmentation of ROI using Connected Component Labeling}

Normally, there are two main categories to segment the image into regions. They are region-based approach (Zhao and Ma, 2012) and edge detection method. In the region based method, seed pixel is selected and based on the some similar criteria all the pixels are selected. 


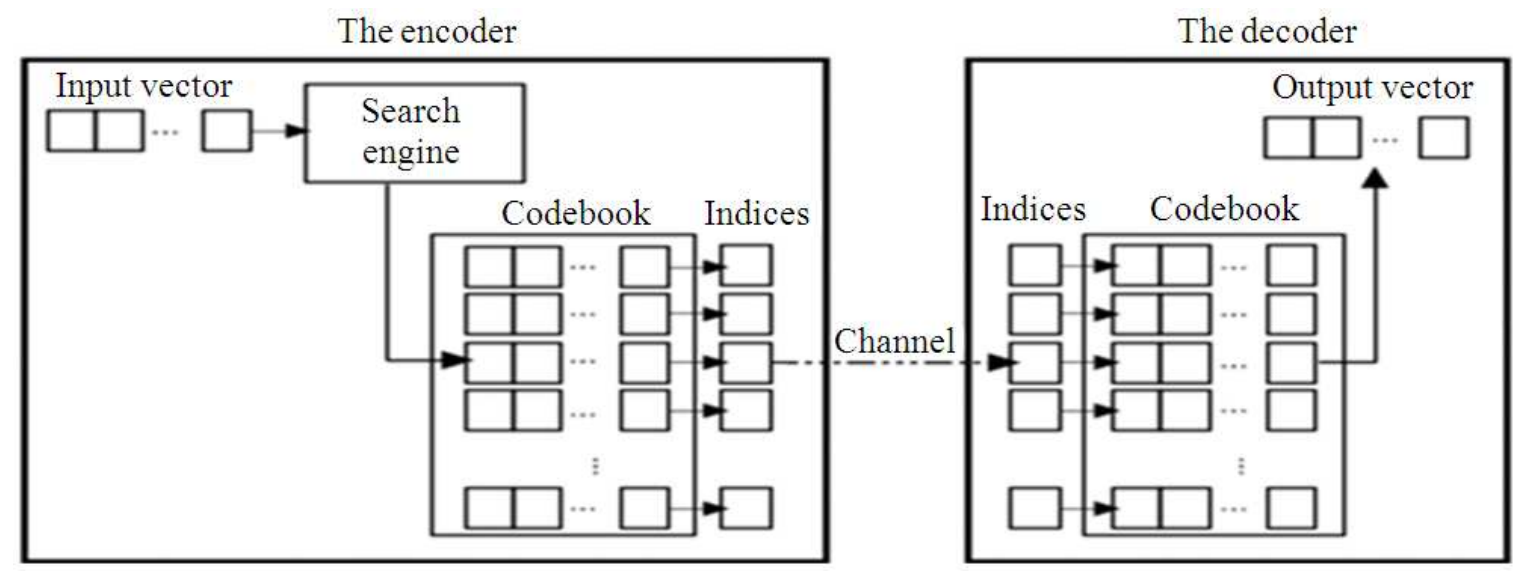

Fig. 1. Block diagram-vector quantization

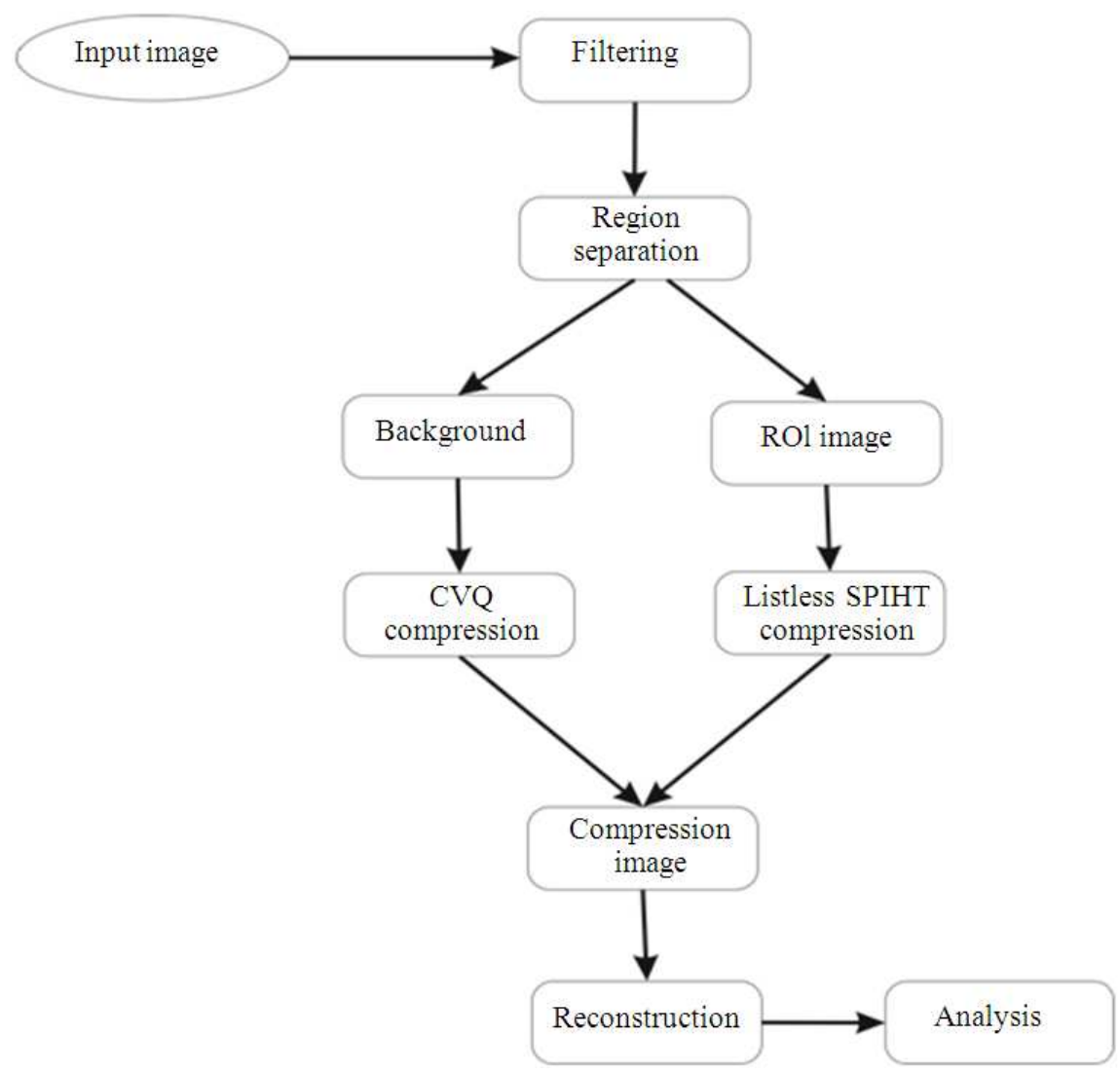

Fig. 2. Proposed hybrid algorithm, (LSPIHT and CVQ)

In the edge based approach, boundary is formed to separate different regions. In our proposed method, connected component labeling method is used to separate the ROI region. Connected-component labeling (Rakhmadi et al., 2010), is used to detect connected regions in digital images and it assigns separate unique label to all points in the component. Here the scanning of pixels starts from left to right and top to bottom. 
Pick any pixel in the image and assign a label and the same label is assigned to any neighbor pixel with the same value of the image function. The process is continued until all the pixels are labeled.

\subsection{Proposed Hybrid Listless (LSPIHT) and CVQ Algorithm}

- Load the brain image as input

- In the preprocessing, Gaussian Filtering is applied to eliminate noise if any

- Using a Connected component labeling method, the ROI and BG are separated

- $\quad$ ROI region is encoded using LSPIHT with high bpp

- $\quad$ BG region is encoded using CVQ with low bpp

- Merge the two encoded regions (ROI and BG) to get the compressed image

- Decode the compressed image to get the reconstructed image

\subsection{Performance Metrics}

The efficiency of the compression algorithm is measured in terms of performance measuring parameters such as Compression Ratio (CR), Peak Signal Noise Ratio (PSNR), bit rate (bpp), Mean Square Error (MSE).

\subsection{Peak Signal to Noise Ratio (PSNR) Equation 4}

$$
\text { PSNR }=10 \log _{10}\left(\frac{255^{2}}{\mathrm{MSE}}\right) \mathrm{dB}
$$

\subsection{Compression Ratio Equation 5 and 6}

$$
\mathrm{CR}=\frac{\text { Size of the compressed image }}{\text { Size of the uncompressed image }}
$$

$$
\text { MSE }=\frac{1}{M \cdot N} \sum_{x=1}^{M} \sum_{y=1}^{N}|f(x, y)-\hat{f}(x, y)|^{2}
$$

\section{RESULTS AND DISCUSSION}

The proposed method is implemented and tested using the image processing software tool, MATLAB 7.9. We considered 50 images of magnetic resonance brain images for the test purpose. The performance parameters bpp, Compression Ratio (CR), Mean Square Error (MSE), Peak Signal to Noise Ratio (PSNR) are given in Table 1 and 2. The parameters are obtained separately for ROI region and the entire image area.

The compression parameters for several compression ratios for ROI region and the entire image is listed in Table 1 and the comparison of bpp with MSE,CR,PSNR are analyzed and plotted in Fig. 3. Table 2 Shows the same performance parameters of compression for Background (BG) image and the complete image and plotted in Fig. 4.

Table 3 shows, our proposed methods outperforms all the existing methods such as Maxshift, CSPIHT, CVQ in terms of PSNR and also plotted in Fig. 5. To show our proposed method is even better than the general compression methods of JPEG2000, SPIHT, it is compared in terms of same PSNR and it is shown in Table 4.

We considered different bitrates for ROI and BG and achieved better compression quality compared to the existing methods. Our proposed method's advantage over all the listed method is more evident as the compression ratio increases.

It is clearly stated in the Table $\mathbf{3}$ and $\mathbf{4}$ and also plotted in Fig. 5 and 6. Our proposed method keeps all the important information needed for the diagnostic purposes with out any loss in the quality. Figure 7 show the results of ROI, BG and the reconstructed image using

\begin{tabular}{|c|c|c|c|c|c|c|c|}
\hline \multirow[b]{2}{*}{ Sl.no } & \multirow[b]{2}{*}{ bpp } & \multicolumn{3}{|c|}{ ROI region } & \multicolumn{3}{|c|}{ Full image } \\
\hline & & CR & MSE & PSNR (dB) & CR & MSE & PSNR (dB) \\
\hline 1. & 1.0000 & 4.910 & 7.820 & 40.48 & 10.78 & 209.319 & 34.426 \\
\hline 2. & 0.5000 & 9.985 & 13.94 & 38.35 & 21.43 & 216.251 & 32.282 \\
\hline 3. & 0.4000 & 12.313 & 16.24 & 37.16 & 25.17 & 224.831 & 30.203 \\
\hline 4. & 0.3000 & 13.021 & 17.01 & 36.28 & 29.26 & 235.283 & 28.391 \\
\hline 5. & 0.2500 & 13.842 & 19.64 & 36.01 & 41.62 & 240.412 & 27.872 \\
\hline 6. & 0.1250 & 19.317 & 21.43 & 35.52 & 124.47 & 311.293 & 25.058 \\
\hline 7. & 0.0625 & 21.148 & 22.72 & 35.14 & 252.31 & 392.417 & 23.835 \\
\hline
\end{tabular}
our proposed hybrid method.

Table 1. LSPIHT- ROI Region and the full image parameters 
Table 2. CVQ- BG region and the full image parameters

\begin{tabular}{llllllll}
\hline & & \multicolumn{3}{c}{ BG region } & & \multicolumn{2}{l}{ Full image } \\
Sl.no & bpp & CR & MSE & PSNR $(\mathrm{dB})$ & CR & MSE & PSNR $(\mathrm{dB})$ \\
\hline 1. & 1.0000 & 10.870 & 172.741 & 32.364 & 11.12 & 189.011 & 36.821 \\
2. & 0.5000 & 20.314 & 179.342 & 30.252 & 15.15 & 198.719 & 35.185 \\
3. & 0.4000 & 22.930 & 182.281 & 29.275 & 18.36 & 202.152 & 33.262 \\
4. & 0.3000 & 31.763 & 190.140 & 28.671 & 20.93 & 211.629 & 33.183 \\
5. & 0.2000 & 50.109 & 196.251 & 27.072 & 23.61 & 227.072 & 30.090 \\
6. & 0.1000 & 102.962 & 201.513 & 26.295 & 27.19 & 235.381 & 28.163 \\
7. & 0.0800 & 129.724 & 225.561 & 26.102 & 30.26 & 241.934 & 27.105 \\
8. & 0.0600 & 169.142 & 268.672 & 24.471 & 48.03 & 271.861 & 25.460 \\
\hline
\end{tabular}

Table 3. Comparison of Maxshift, CSPIHT, CVQ and our proposed hybrid method, in terms of PSNR (dB)

\begin{tabular}{|c|c|c|c|c|c|}
\hline \multirow[b]{2}{*}{ bpp } & \multirow[b]{2}{*}{$\mathrm{CR}$} & \multicolumn{4}{|c|}{ PSNR (dB) } \\
\hline & & Maxshift & CSPIHT & CVQ & Proposed \\
\hline 1.0000 & 12.01 & 42.73 & 40.27 & 42.99 & 43.16 \\
\hline 0.5000 & 35.19 & 39.42 & 38.31 & 41.04 & 42.94 \\
\hline 0.2500 & 68.36 & 36.10 & 35.81 & 38.26 & 42.31 \\
\hline 0.1250 & 128.02 & 30.81 & 30.95 & 35.37 & 37.63 \\
\hline 0.0625 & 256.13 & 27.43 & 27.74 & 32.68 & 35.26 \\
\hline
\end{tabular}

Table 4. Comparison of JPEG2000,SPIHT and the proposed, in terms of PSNR (dB

\begin{tabular}{llll}
\hline & PSNR $(\mathrm{dB})$ & & \\
$\mathrm{bpp}$ & $\mathrm{JPEG} 2000$ & SPIHT & Proposed \\
\hline 1.0000 & 30.56 & 39.49 & 43.16 \\
0.5000 & 28.73 & 37.07 & 42.94 \\
0.2500 & 27.15 & 32.73 & 42.31 \\
0.1250 & 25.01 & 28.05 & 37.63 \\
0.0625 & 24.72 & 25.14 & 35.26 \\
\hline
\end{tabular}

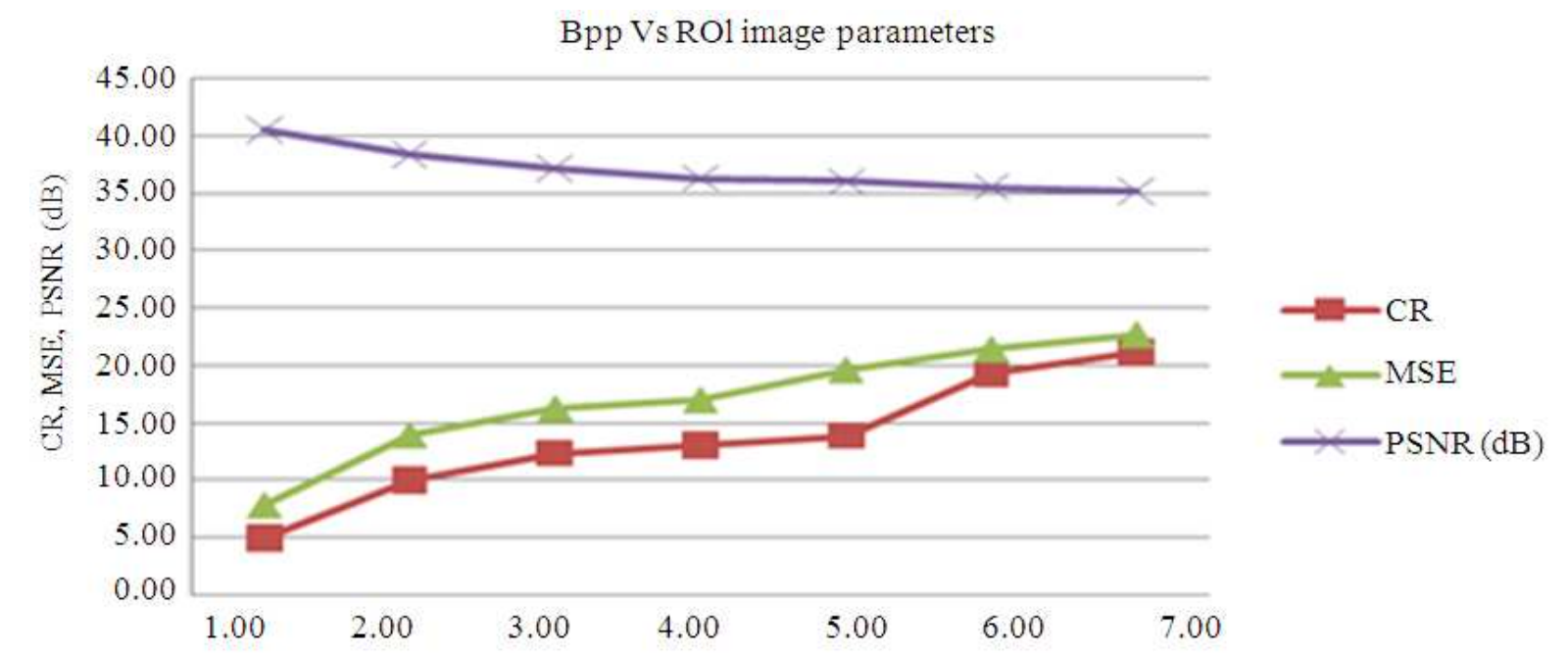

Fig. 3. Comparison of compression parameters-bpp Vs. MSE, CR, PSNR for ROI region at different bit rates (bpp) 
Sridevi, S. and V.R. Vijayakumar / Journal of Computer Science 9 (9): 1181-1189, 2013

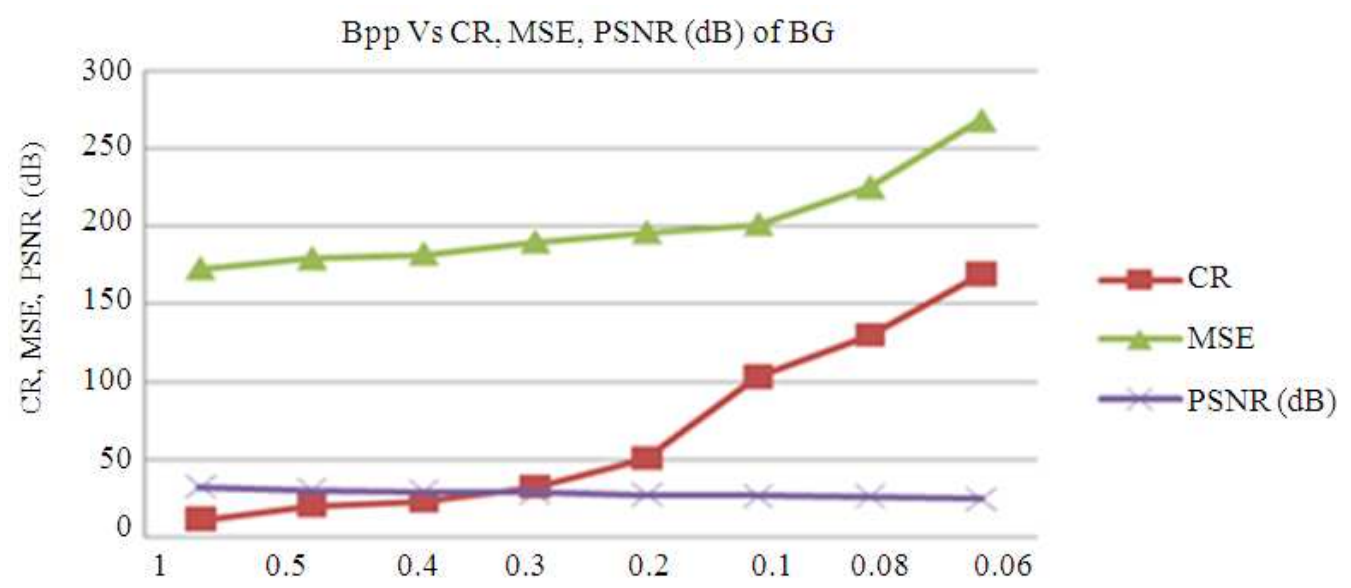

Fig. 4. Comparison of compression parameters-bpp Vs MSE, CR, PSNR for BG at different bit rates (bpp)

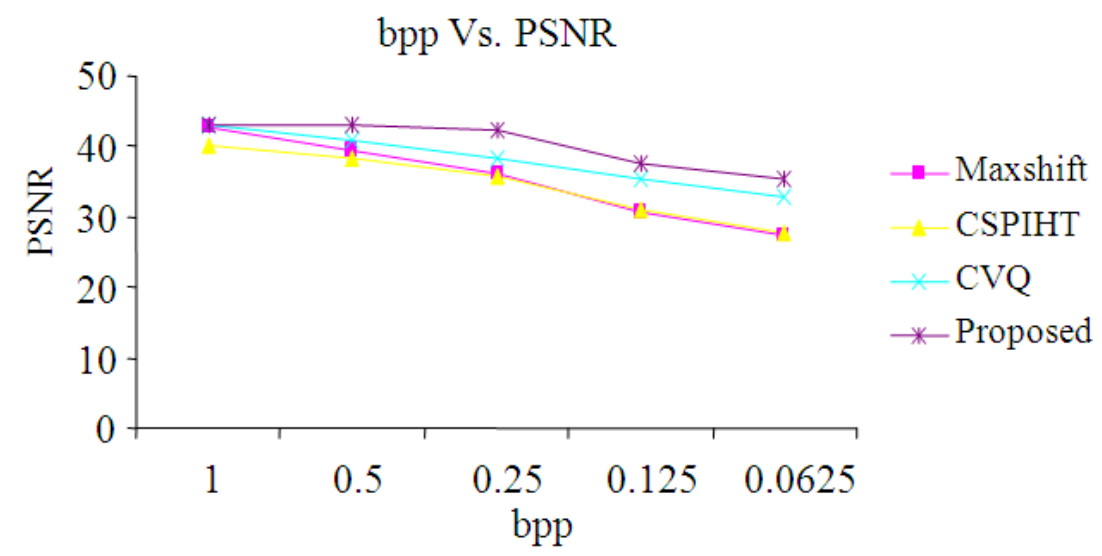

Fig. 5. Comparison of bpp Vs PSNR (dB) for Maxshift, CSPIHT, CVQ and our proposed method

bpp Vs. PSNR (dB)

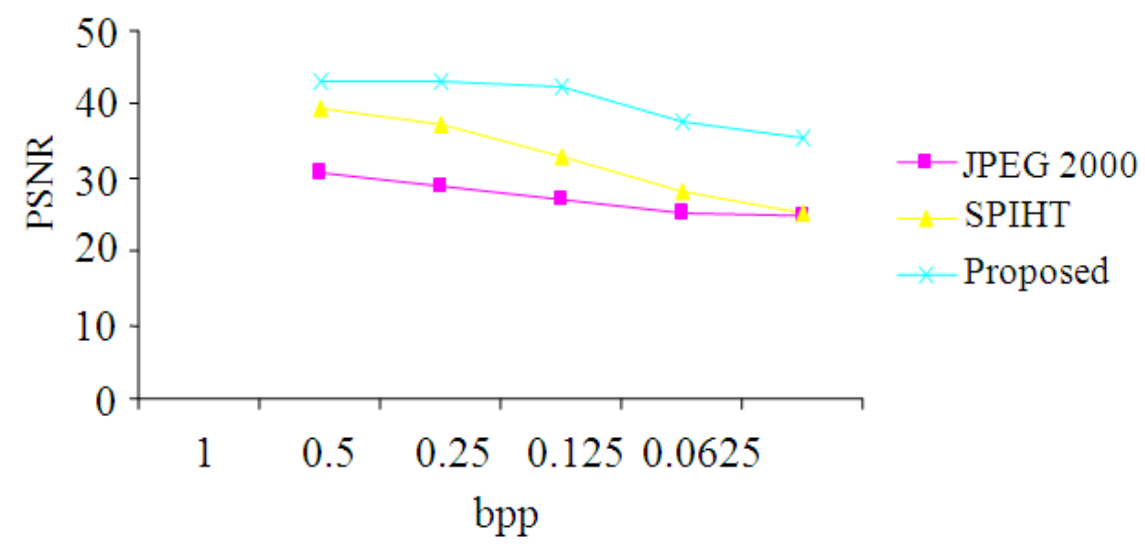

Fig. 6. Comparison of bpp Vs PSNR (dB) for JPEG2000, SPIHT and our proposed hybrid LSPIHT and CVQ 


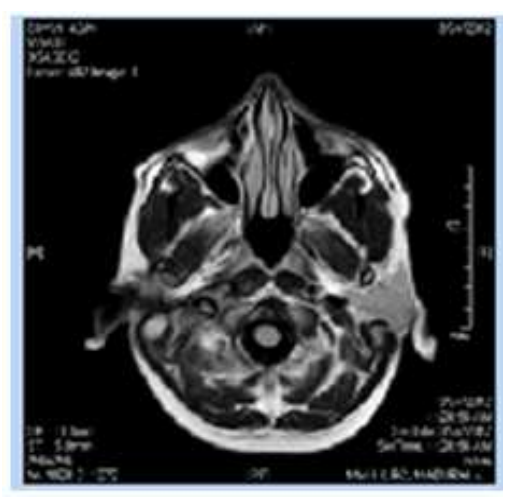

(a)

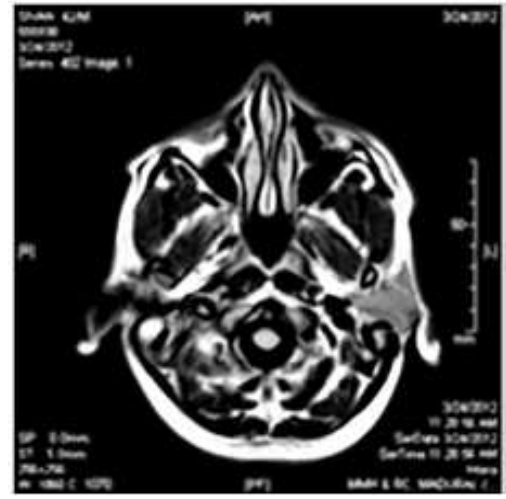

(b)

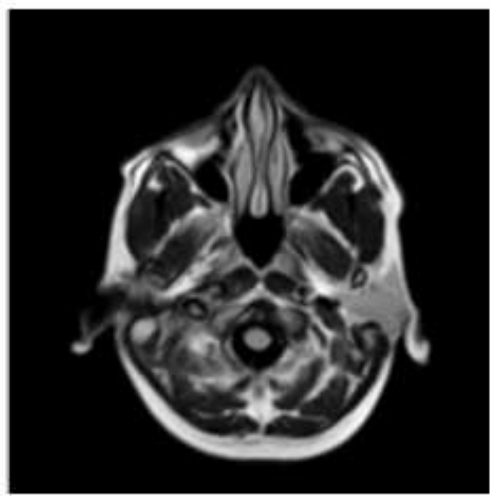

(c)

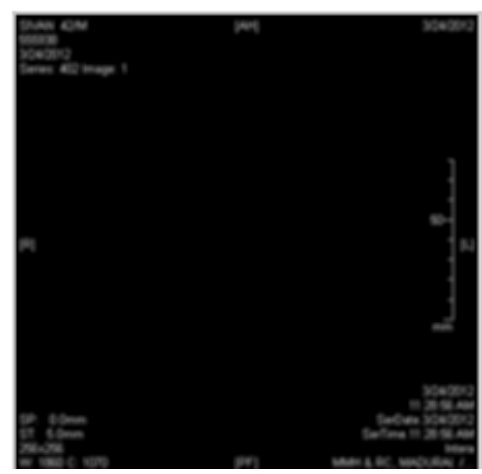

(d)

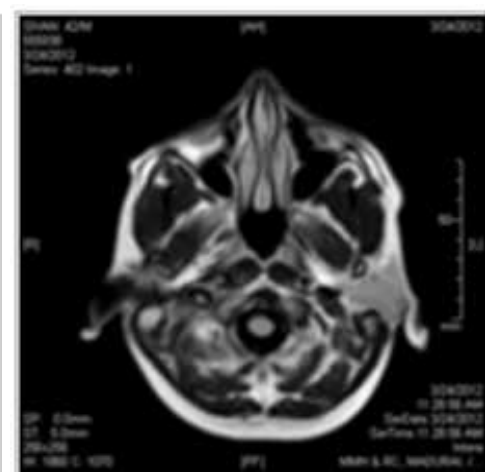

(e)

Fig. 7. (a-e) Original image and the separation of ROI and BG, final reconstructed image using LSPIHT and CVQ method

\section{CONCLUSION}

In order to obtain high compression ratio and good image quality, this study presented a new hybrid algorithm based on ROI technique. With the help of connected component labeling method, the segmentation of $\mathrm{ROI}$ region and $\mathrm{BG}$ regions are separated and the ROI is encoded separately using Listless SPIHT and the BG is encoded using CVQ. Finally, the two regions are merged together to get the reconstructed image. From the results obtained by our method, show the improved performance in terms of performance parameters like CR, MSE, PSNR (dB) and the same are compared with the existing methods. Our method superiority is shown even better when comparing it with the traditional methods of JPEK2K and SPIHT. We achieved better reconstructed image and the same is judged for visual quality on the basis of the Human Visual System (HVS). So, finally, we conclude that our proposed hybrid method is a good choice for the compression of medical images and it can also maintain high quality of reconstructed images without any loss of important information for diagnosis. Using our method, we strongly believe that our method can reduce the storage cost and transmission time of medical images. In future, we can implement our method to compress the 3D medical data which are produced every day.

\section{ACKNOWLEDGEMENT}

The researchers wish to thank Dr. Amba Bhavani, Amba Clinic and Dr. Devaki from Devaki Cancer Institute, Madurai for the provision of medical image data sets used in this study. I am so thankful to my supervisor, for providing constant support and the guidelines given for my research work.

\section{REFERENCES}

Alam, M. and E. Khan, 2012. Listless Highly scalable set parititioning in hierarchical trees coding for transmission of image over heterogenous networks. Int. J. Comput. Networ. Wireless Mobile Commun. 
Ansari, M.A. and R.S. Anand, 2009. Context based medical image compression for ultrasound images with contextual set partitioning in hierarchical trees algorithm. Adv. Eng. Soft., 40: 487-496. DOI: 10.1016/j.advengsoft.2008.08.004

Bartrina-Rapesta, J., J. Serra-Sagristà and F. Aulí-Llinàs, 2011. JPEG2000 ROI coding through component priority for digital mammography. Comput. Vis. Image Understand., 115: 59-68. DOI: 10.1016/j.cviu.2010.09.008

Gonzalez, R.C. and R.E. Woods, 2009. Digital Image Processing. 1st Edn., Pearson Education India, India, ISBN-10: 8131726959, pp: 954.

Grailu, H., R. Soltani and M. Akrami, 2013. ROI soccer video compression using wavelet-domain block matching and EBCOT coding techniques in very low bit rate communication. Proceedings of the 1st Iranian Conference on Pattern Recognition and Image Analysis, Mar. 6-8, IEEE Xplore Press, Birjand, pp: 1-4. DOI: 10.1109/PRIA.2013.6528430

Hosseini, S.M. and A.R. Naghsh-Nilchi, 2012. Medical ultrasound image compression using Contextual vector quantization. Comput. Biol. Med., 42: 743750. DOI: 10.1016/j.compbiomed.2012.04.006

Lim, N.K., D.Y. Kim and H. Lee, 2010. Interactive progressive image transmission for realtime applications. IEEE Trans. Consumer Electron., 56: 2438-2444. DOI: 10.1109/TCE.2010.5681125

Lu, T.C. and C.Y. Chang, 2010. A survey of VQ codebook generation. J. Inform. Hid. Multimed. Signal Proc., 1: 190-203.

Noma, A., A.B.V. Graciano, R.M. Cesar Jr, L.A. Consularo and I. Bloch, 2012. Interactive image segmentation by matching attributed relational graphs, Patt. Recogn., 45: 1159-1179. DOI: 10.1016/j.patcog.2011.08.017
Rakhmadi, A., N.Z.S. Othman, A. Bade, M.S.M. Rahim and I.M. Amin, 2010. Connected Component labeling using component neighbours-scan labeling approach. J. Comput. Sci., 6: 1099-1107. DOI: 10.3844/jcssp.2010.1099.1107

Sanchez, V., R. Abugharbieh and P. Nasiopoulos, 2010. 3-D scalable medical image compression with optimized volume of interest coding. IEEE Trans. Med. Imag., 29: 1808-1820. DOI: 10.1109/TMI.2010.2052628

Selvi, G.U.V. and R. Nadarajan, 2012. Coronary angiogram video compression using wavelet-based contourlet transform and region-of-interest technique. IET Image Process., 6: 1049-1056. DOI: 10.1049/iet-ipr.2011.0284

Seo, Y.G., K.S. Kim and S.G. Kim, 2010. Generating a fast ROI mask using approximate division of code blocks In JPEG2000. Int. J. Wavelets Multiresolut Inform. Process. DOI: 10.1142/S0219691310003584

Singh, P. and P. Singh, 2012. Implementation of SPIHT and WDR algorithms for natural and artificial images using wavelets. Proceedings of the 4th International Conference on Computational Intelligence and Communication Networks, Nov. 35, IEEE Xplore Press, Mathura, pp: 359-363. DOI: 10.1109/CICN.2012.132

Thorat, C.G. and B.D. Jadhav, 2010. A blind digital watermark technique for color image based on integer wavelet transform and SIFT. Proc. Comput. Sci., 2: 236-241. DOI: 10.1016/J.PROCS.2010.11.030

Zhao, R. and Y. Ma, 2012. A region segmentation method for region-oriented image compression. Neurocomputing, 85: 45-52. DOI: 10.1016/j.neucom.2012.01.007 